\title{
BANACH SPACES WHICH ARE $M$-IDEALS IN THEIR BIDUALS
}

\author{
BY
}

\author{
PETER HARMAND AND ÅSVALD LIMA
}

\begin{abstract}
We investigate Banach spaces $X$ such that $X$ is an $M$-ideal in $X^{* *}$. Subspaces, quotients and $c_{0}$-sums of spaces which are $M$-ideals in their biduals are again of this type. A nonreflexive space $X$ which is an $M$-ideal in $X^{* *}$ contains a copy of $c_{0}$. Recently Lima has shown that if $K(X)$ is an $M$-ideal in $L(X)$ then $X$ is an $M$-ideal in $X^{* *}$. Here we show that if $X$ is reflexive and $K(X)$ is an $M$-ideal in $L(X)$, then $K(X)^{* *}$ is isometric to $L(X)$, i.e. $K(X)$ is an $M$-ideal in its bidual. Moreover, for real such spaces, we show that $K(X)$ contains a proper $M$-ideal if and only if $X$ or $X^{*}$ contains a proper $M$-ideal.
\end{abstract}

1. Introduction. The object of this paper is to investigate Banach spaces $X$, which are $M$-ideals in their bidual $X^{* *}$. Previous investigations of the question, whether the space of compact operators on $X$ is an $M$-ideal in the space of all bounded operators, and the general study of the nature of the embedding of $X$ into $X^{* *}$ have led us to consider these spaces.

In $\S 2$ we show some general results concerning $M$-structure, which are perhaps folklore, but which do not appear in the literature.

In $\$ 3$ we start to study the properties of spaces which are $M$-ideals in their biduals. We show that subspaces and quotients of spaces, which are $M$-ideals in their biduals, have the same property. The proof of this result is based upon the fact that $X$ is an $M$-ideal in $X^{* *}$ if and only if the natural projection from $X^{* * *}$ onto $X^{*}$ is an $L$-projection. Using local reflexivity we show that if $X$ is an $M$-ideal in $X^{* *}$ and $X$ is nonreflexive, then $X$ contains almost isometric copies of $c_{0}$. From this it follows that subspaces and quotients of such papers which are isomorphic to dual spaces are reflexive.

$M$-structure of spaces which are $M$-ideals in their biduals are studied in $\S 4$. Here we show that if $X$ is an $M$-ideal in $X^{* *}$ without proper $M$-summands and $J \neq\{0\}$ is an $M$-ideal in $X^{* *}$, then $X \subset J$.

In $\S 5$ we treat the relation between spaces which are $M$-ideals in their biduals and spaces of compact operators. In [12] Lima proved that if $K(X)$ is an $M$-ideal in $L(X)$, then $X$ is an $M$-ideal in $X^{* *}$ and $X^{*}$ has the RNP. Here we show that if $X$ is reflexive and $K(X)$ is an $M$-ideal in $L(X)$, then $K(X)^{* *}$ is isometric to $L(X)$, i.e. $K(X)$ is an $M$-ideal in its bidual. Moreover, if $K(X)$ is an $M$-ideal in its bidual, then $X$ is reflexive. We prove that for real spaces $K(X, Y)$ contains a proper $M$-summand

Received by the editors September 10, 1982 and, in revised form, May 4, 1983.

1980 Mathematics Subject Classification. Primary 46B20; Secondary 46B10, 47D15.

$K e y$ words and phrases. $M$-ideal, bidual, $M$-structure, spaces of compact operators.

1984 American Mathematical Society $0025-5726 / 84 \$ 1.00+\$ .25$ per page 
if and only if $Y$ or $X^{*}$ contains a proper $M$-summand. For $X$ reflexive this means that $K(X)$ contains a proper $M$-ideal if and only if $X$ or $X^{*}$ contains a proper $M$-ideal.

Let us fix some notation. A closed subspace $J$ of a Banach space $X$ is called an $L^{p}$-summand $(1 \leqslant p<\infty)$ (resp. $M$-summand) if there exists a closed subspace $J^{\perp}$ such that $X=J \oplus J^{\perp}$ and

$$
\|x+y\|^{p}=\|x\|^{p}+\|y\|^{p} \quad \text { for } x \in J, y \in J^{\perp}
$$

(resp. $\|x+y\|=\max \{\|x\|,\|y\|\}$ for $x \in J, y \in J^{\perp}$ ). $L^{p_{-}}$and $M$-summands are equivalently characterized as ranges of $L^{p}$ - and $M$-projections, which are defined in an obvious way. A closed subspace $J$ of $X$ is called an $M$-ideal if its annihilator $J^{0}$ in $X^{*}$ is an $L$-summand (in the case $p=1$, we suppress the $p$ ). $M$-ideals can also be described by intersection properties of balls. For more information we refer to [2].

We denote by $K(X, Y)(K(X)$ in the case $X=Y)$ the space of compact operators from $X$ to $Y$ and by $L(X, Y)(L(X)$ if $X=Y)$ the space of all bounded operators from $X$ to $Y . B_{X}(a, r)$ is the closed ball with center $a$ and radius $r$ in $X$ (we omit the $X$ if the space is clear from the context). $B_{X}$ is the unit ball of $X . X \simeq Y(X \cong Y)$ means that these spaces are isomorphic (isometrically isomorphic).

We write $X \oplus_{\infty} Y$ or $X \oplus_{1} Y$ if the norm on the direct sum of $X$ and $Y$ is the maximum- or the sum-norm.

The first named author wishes to thank E. Behrends and R. Evans for many discussions. Some of the first results are due to R. Evans.

2. Preliminaries about $M$-structure. The first result shows that $M$-projections ( $L$-projections) are uniquely determined among contractive projections by their ranges (kernels).

2.1. Proposition. Let $X$ be a Banach space.

(a) If $E$ is an $M$-projection in $X$ and $P$ is a contractive projection with the same range, then $P=E$.

(b) If $E$ is an L-projection in $X$ and $P$ is a contractive projection with the same kernel, then $P=E$.

Proof. ad(a). It suffices to prove ker $E=\operatorname{ker} P$.

" $\subset$ ". Let $x \in \operatorname{ker} E$ with $\|x\|=1$ be given. Suppose $P x \neq 0$. For $y:=x+$ $P x /\|P x\| \in \operatorname{ker} E \oplus_{\infty}$ im $E$ we then have $\|y\|=1$. Hence

$$
\|P y\|=\left(1+\frac{1}{\|P x\|}\right)\|P x\|=\|P x\|+1>1
$$

which contradicts $\|P\| \leqslant 1$.

" $\supset$ ". Let $x \in \operatorname{ker} P$ be given. For $y:=x-E x$ we have $y \in \operatorname{ker} E \subset \operatorname{ker} P$. Hence

$$
0=P y=P x-P E x=-E x, \text { i.e. } x \in \operatorname{ker} E .
$$

ad(b). This follows from (a) by dualising.

2.2. Corollary. Let $J$ be an $M$-ideal in $X$. If $J$ is the range of a contractive projection, then $J$ is an M-summand. 
Proof. Let $P$ be the projection onto $J,\|P\| \leqslant 1$. Then $P^{*}$ is a contractive projection with ker $P^{*}=J^{0}$. Because $J^{0}$ is an $L$-summand, $P^{*}$ must be the corresponding $L$-projection (Proposition 2.1(b)). But then $P$ is an $M$-projection [2, Proposition 1.5].

2.3. Proposition. Let $X$ be a Banach space and let $J$ be an M-ideal in $X$. If $X=K \oplus_{\infty} L$ then $J=(K \cap J) \oplus_{\infty}(L \cap J)$.

Proof. Let $E$ be the $M$-projection onto $K$. Obviously it suffices to show $E J \subset J$. By a simple Hahn-Banach argument this in turn is equivalent to $E^{*} J^{0} \subset J^{0}$. Now $E^{*}$ is an $L$-projection [2, Proposition 1.5] and $J^{0}$ is an $L$-summand. Let $P$ be the $L$-projection onto $j^{0}$. The commutativity of $L$-projections [2, Proposition 1.7] then gives $E^{*} J^{0}=E^{*} P X=P E^{*} X^{*} \subset P X^{*}=J^{0}$.

3. Properties of spaces which are $M$-ideals in their biduals. In the sequel we will frequently identify $i_{X}(X)$ and $X$. Since $M$ - and $L^{p}$-summands $(1<p<\infty)$ in dual spaces are $w^{*}$-closed [2, Theorem 5.6; 3, Lemma 2.9], a nonreflexive Banach space cannot be an $M$ - or $L^{p}$-summand $(1<p<\infty)$ in its bidual.

3.1. Proposition. Let $X$ be a Banach space. The following statements are equivalent.

(a) $X$ is an $M$-ideal in $X^{* *}$.

(b) The natural projection from $X^{* * *}$ onto $i_{X^{*}}\left(X^{*}\right)$ is an L-projection.

Proof. Note that according to the definition $X$ is an $M$-ideal in $X^{* *}$ if and only if $i_{X}(X)^{0}$ is an $L$-summand in $X^{* * *}$.

(a) $\Rightarrow$ (b). The $L$-projection $E$ onto the $L$-summand complementary for $i_{X}(X)^{0}$ has the kernel $i_{X}(X)^{0}$. Because this is also the kernel of the natural projection, Proposition 2.1(b) proves this half of the equivalence.

(b) $\Rightarrow$ (a). Trivial.

\subsection{Corollary. If $X$ is an $M$-ideal in $X^{* *}$, then $X^{*}$ is an L-summand in $X^{* * *}$.}

See the remarks following Theorem 4.4 for the converse.

3.3. EXAMPLES. Only nonreflexive Banach spaces are interesting.

(a) $c_{0}, K(H)$ with $H$ a Hilbert space, and $K\left(l_{p}, l_{q}\right)$ with $1<p \leqslant q<\infty$ are nonreflexive $M$-ideals in their biduals. ( $c_{0}$ and $K(H)$ are two-sided, closed ideals in their biduals $l_{\infty}$ and $L(H)$; with [20, Theorem 5.3] they are $M$-ideals. Using tensor products one easily sees that: "If $X$ and $Y$ are reflexive spaces with the approximation property, then $K(X, Y)^{* *} \cong L(X, Y)$ " and " $K\left(l_{p}, l_{q}\right)$ is nonreflexive for $1<p$ $\leqslant q<\infty$ " (cf. [4, Chapter VIII]); Theorem 9 of [18] then gives the desired result. See also [13].)

(b) $L^{1}(S, \Sigma, \mu)$ is an $L$-summand in its bidual. (This is immediate from the fact that these spaces are projection bands in their biduals, which are again AL-spaces.) More general is the following: Let $A$ be a $W^{*}$-algebra. Then $A_{*}$ (the predual of $A$ ) is an $L$-summand in its bidual [23, Theorem 3]. In [1] Ando has shown that $L^{1} / H_{0}^{1}$ is an $L$-summand in its bidual. 
The next theorem establishes the hereditary properties of spaces which are $M$-ideals in their biduals.

3.4. THEOREM. If $X$ is an $M$-ideal in $X^{* *}$, then we have:

(a) Subspaces of $X$ are $M$-ideals in their biduals.

(b) Quotients of $X$ are $M$-ideals in their biduals.

(c) $c_{0}$-sums of spaces which are M-ideals in their biduals are M-ideals in their biduals.

Proof. ad(a). Let $M$ be a closed subspace of $X$. By Proposition 3.1 we have to show that the natural projection $P_{M^{*}}$ of $M^{* * *}$ onto $i_{M^{*}}\left(M^{*}\right)$ is an $L$-projection. Recall the following two facts: " $P_{M^{*}} T^{* * *}=T^{* * *} P_{X^{*}}$ where $T: M \rightarrow X$ denotes the inclusion mapping", " $T^{* * *}$ is a quotient mapping, i.e. given $m \in M^{* * *}$ and $\varepsilon>0$ one can find $x \in X^{* * *}$ with $T^{* * *} x=m$ and $\|x\| \leqslant\|m\|+\varepsilon$ ". By assumption $P_{X^{*}}$ is an $L$-projection. Let $m, \varepsilon$, and $x$ be as above. Then we have

$$
\begin{aligned}
\|m\| & \leqslant\left\|P_{M^{*}} m\right\|+\left\|m-P_{M^{*}} m\right\|=\left\|P_{M^{*}} T^{* * *} x\right\|+\left\|T^{* * *} x-P_{M^{*}} T^{* * *} x\right\| \\
& =\left\|T^{* * *} P_{X^{*}} x\right\|+\left\|T^{* * *} x-T^{* * *} P_{X^{*}} x\right\| \\
& \leqslant\left\|T^{* * *}\right\|\left(\left\|P_{X^{*}} x\right\|+\left\|x-P_{X^{*}} x\right\|\right)=\|x\| \leqslant\|m\|+\varepsilon
\end{aligned}
$$

which shows that $P_{M^{*}}$ is an $L$-projection.

ad(b). Replace $T$ by the canonical map $\omega: X \rightarrow X / M$. Then the proof is similar to that of (a). (Actually it is easier, because $\omega^{* * *}$ is isometric.)

$\operatorname{ad}(c)$. This is straightforward.

Remarks. (1) Using the identification $(X / M)^{*} \cong M^{0}$ and Proposition 1.5(2) of [14] one can also give a more direct proof of Theorem 3.4(b) using the intersection property of $M$-ideals in the form of [11].

(2) One easily sees that being an $M$-ideal in its bidual is not a 3-space-property.

(3) Although the nature of the objects in $M$-structure theory is purely isometric, it easily follows from the characterization by the intersection property that if $X$ is an $M$-ideal in $X^{* *}$ and $d(X, Y)=1$ then $Y$ is an $M$-ideal in $Y^{* *} . d(X, Y)$ denotes the Banach-Mazur distance.

We shall now show that nonreflexive spaces which are $M$-ideals in their biduals contain almost isometric copies of $c_{0}$ and hence, that they are not isomorphic to dual spaces. We write the proof of Theorem 3.5 for real spaces only. The proof is easily extended to the complex case.

3.5. TheOrem. Let $X$ be nonreflexive and an $M$-ideal in $X^{* *}$. Then $X$ contains almost isometric copies of $c_{0}$.

Proof. Let $\varepsilon>0$ and choose $x_{1} \in X$ and $y \in X^{* *}$ such that $1=\left\|x_{1}\right\|=d(y, X)$ and $\|y\|<1+\varepsilon$. We shall show by induction that there exists a sequence $\left(x_{n}\right)_{n=1}^{\infty}$ in $X$ such that:

(i) $\left\|x_{n}\right\| \geqslant 1-2 \varepsilon$ for all $n$.

(ii) $\left\|x_{1} \pm x_{2} \pm \cdots \pm x_{n}\right\|<1+2 \varepsilon-2 \varepsilon / 2^{n}$ for all $n$ and all choice of signs. 
Assume we have found $x_{1}, \ldots, x_{n}$ as above. Since $X$ is an $M$-ideal in $X^{* *}$, there exists a $z_{n} \in X$ such that $[\mathbf{1 1}]$

$$
\left\|\left(y-z_{n}\right) \pm x_{1} \pm x_{2} \pm \cdots \pm x_{n}\right\|<1+2 \varepsilon-\frac{2 \varepsilon}{2^{n}}
$$

for all choice of signs. Let $E_{n}=\operatorname{span}\left(y, z_{n}, x_{1}, \ldots, x_{n}\right)$. By the principle of local reflexivity [15], there exists an operator $T_{n}: E_{n} \rightarrow X$ such that for all $x \in E_{n}$

$$
\left(1-\partial_{n}\right)\|x\| \leqslant\left\|T_{n} x\right\| \leqslant\left(1+\partial_{n}\right)\|x\|
$$

and $T_{n} x=x$ if $x \in E_{n} \cap X$. Here we choose $\partial_{n}>0$ such that

$$
\left(1+\partial_{n}\right)\left(1+2 \varepsilon-\frac{2 \varepsilon}{2^{n}}\right)<\left(1+2 \varepsilon-\frac{\varepsilon}{2^{n}}\right)=\left(1+2 \varepsilon-\frac{2 \varepsilon}{2^{n+1}}\right)
$$

Put $x_{n+1}=T_{n}\left(y-z_{n}\right)$. Then we have

$$
\begin{aligned}
\left\|x_{1} \pm x_{2} \pm \cdots \pm x_{n} \pm x_{n+1}\right\| & =\left\|T_{n}\left(y-z_{n} \pm x_{1} \pm x_{2} \pm \cdots \pm x_{n}\right)\right\| \\
& \leqslant\left(1+\partial_{n}\right)\left(1+2 \varepsilon-\frac{2 \varepsilon}{2^{n}}\right)<1+2 \varepsilon-\frac{2 \varepsilon}{2^{n+1}} .
\end{aligned}
$$

Moreover $\left(1-\partial_{n}\right)\left\|y-z_{n}\right\| \leqslant\left\|x_{n+1}\right\|$ and since $z_{n} \in X$ we have $\left\|y-z_{n}\right\| \geqslant$ $d(y, X)=1$. Thus we get $\left\|x_{n+1}\right\| \geqslant 1-\partial_{n} \geqslant 1-2 \varepsilon$ for $\partial_{n}$ sufficiently small.

Now we define an operator $T: c_{0} \rightarrow X$ by $T\left(\left(a_{n}\right)\right)=\sum_{n} a_{n} x_{n}$. It is straightforward to show that

$$
(1-6 \varepsilon)\|x\| \leqslant\|T x\| \leqslant(1+2 \varepsilon)\|x\| \text { for all } x \in c_{0} .
$$

The first corollary from this theorem collects some simple, but interesting consequences. The second is more substantial, because it says that nonreflexive spaces which are $M$-ideals in their biduals are far from being dual spaces. Note that an alternative proof of Corollary 3.7 could be given, using (c) of Corollary 3.6 and Theorem $23 \mathrm{~B}$ in [10].

3.6. Corollary. Let $X$ be a nonreflexive space which is an $M$-ideal in $X^{* *}$.

(a) $X$ is not weakly sequentially complete.

(b) $X$ does not have the Radon-Nikodym property.

(c) $X$ is not complemented in $X^{* *}$.

(d) $X^{* *} / X$ is nonseparable.

Proof. (a) and (b) follow from the fact that $c_{0}$ is not wsc and does not have RNP and that these properties are hereditary for subspaces.

(c) Since $X$ is an $M$-ideal in $X^{* *}, X$ contains a subspace isomorphic to $c_{0}$. By a result of Rosenthal [16, Theorem 1.3], if $X$ is complemented in $X^{* *}$, then $X$ contains a subspace isomorphic to $l_{\infty}$. Hence $X$ also contains $l_{1}$. But this is impossible since $X^{*}$ has the RNP [14]. Thus $X$ is not complemented in $X^{* *}$.

(d) follows from the embedding $l_{\propto} / c_{0} \rightarrow X^{* *} / X$ (there we use the result "if $J$ is isomorphic to a subspace of $X$, then $J^{* *} / J$ is isomorphic to a subspace of $X^{* *} / X^{\text {" }}$ which is not difficult to prove).

3.7. Corollary. Let $X$ be an M-ideal in $X^{* *}$ and let $Y$ be a closed subspace or a quotient space of $X$. If $Y$ is isomorphic to a dual space, then $Y$ is reflexive. 
Proof. By Theorem 3.4(a) or (b) $Y$ is an $M$-ideal in $Y^{* *}$. Let $Y \simeq Z^{*}$ and assume for contradiction that $Y$ is not reflexive. Then $Y$, hence $Z^{*}$, contains $c_{0}$ and by Proposition I2.e.8 in [15] also $l_{x} \subset Y$. But then $Y^{*}$ does not have the RNP $\left(l_{1} \hookrightarrow l_{x} \hookrightarrow Y\right.$ and $l_{1}^{*}$ is not separable). By Theorem 2.6 in Lima [14] we get that $Y$ is not an $M$-ideal in $Y^{* *}$.

4. $M$-structure of spaces which are $M$-ideals in their biduals. We investigate the $M$-ideals and the centralizer of spaces which are $M$-ideals in their biduals. In Theorem 4.4 we show that if $X$ is an $M$-ideal in $X^{* *}$, then either $X$ contains a proper $M$-summand or else $X$ is contained in every proper $M$-ideal of $X^{* *}$.

4.1. Theorem. Let $X$ be an $M$-ideal in $X^{* *}$ and let $J$ be a closed subspace of $X$. If $J$ is an $M$-ideal in $X$, then $J$ is an $M$-summand in $X$.

Proof. By assumption $J^{00}$ is an $M$-summand in $X^{* *}$. Using the fact that $i_{X}(J)=J^{(0)} \cap i_{X}(X)$, which is a simple application of the Hahn-Banach theorem, and Proposition 2.3 one arrives at the desired result.

The next result is not only useful for proving Theorem 4.3, but is of some interest in its own right.

4.2. Proposition. Let $X$ be an $M$-ideal in $X^{* *}$. Then every isometric isomorphism of $X^{* *}$ is the bitranspose of an isometric isomorphism of $X$.

Proof. Let $I: X^{* *} \rightarrow X^{* *}$ be the isometric isomorphism. From [14, Theorem 2.6] we know that $X^{*}$ has the RNP. A result of Godefroy [7, p. 103] then gives an isometric isomorphism $J: X^{*} \rightarrow X^{*}$ with $J^{*}=I$. We only have to show $J^{*} X \subset X$, because then $\left(\left.I\right|_{X}\right)^{*}=J$. By a simple Hahn-Banach argument this inclusion is equivalent to $I^{*} X^{0} \subset X^{0}$. To prove this, it suffices to show $I^{*} B_{X^{0}} \subset B_{X^{0}}$.

Because of the $w^{*}$-continuity of $I^{*}$ and the fact that $B_{X^{0}}=\overline{\operatorname{conv}}^{{ }^{* *}}$ ext $B_{X^{0}}$, we see that it is enough to show that every extreme point $f \in B_{X^{0}}$ is mapped by $I^{*}$ into $B_{X^{0}}$. But $I^{*} f$ is an extreme point in $B_{X^{* * *}}$ and, because $X^{* * *}=X^{*} \oplus_{1} X^{0}$, it therefore can only lie in $B_{X^{0}}$ or $B_{X^{*}}$. In the second case there exist a $g \in X^{*}$ with $J^{* *} f=I^{*} f=J g$ $=J^{* *} g$, which contradicts the fact that $J^{* *}$ is one-to-one.

REMARK. G. Godefroy has informed us that he has independently proved Proposition 4.2 in a forthcoming paper.

For the following theorem we need some further concepts of $M$-structure: We denote by $P_{M}(X)\left(P_{L}(X)\right)$ the collection of all $M$ - $(L$-)projections in a Banach space $X$ and their closed linear spans in $L(X)$ by $C_{x}(X)(C(X))$. For a definition of $Z(X)$, the centralizer of $X$, we refer to [2, p. $53 \mathrm{ff}]$. Here we need only the fact $C_{x}(X) \subset Z(X)$ with equality for dual spaces [2, Theorem 5.9].

\subsection{Theorem. Let $X$ be an $M$-ideal in $X^{* *}$. Then $Z(X) \cong Z\left(X^{* *}\right)$.}

Proof. Clearly the operator $A: L(X) \rightarrow L\left(X^{* *}\right)$ defined by $T \rightarrow T^{* *}$ gives an isometric injection from $P_{M}(X)$ into $P_{M}\left(X^{* *}\right)$. If $P \in P_{M}\left(X^{* *}\right)$ then $I=2 P-$ id is an isometric isomorphism of $X^{* *}$, hence bitranspose by Proposition 4.2. It easily follows that $P$ is the bitranspose of an $M$-projection in $X$, i.e.

$$
P_{M}\left(X^{* *}\right)=A\left(P_{M}(X)\right) \subset A\left(C_{\infty}(X)\right) .
$$


As the right-hand side is a closed linear space, this gives

$$
Z\left(X^{* *}\right)=C_{\infty}\left(X^{* *}\right) \subset A\left(C_{\infty}(X)\right) \subset A(Z(X)) .
$$

Together with the opposite inclusion $A(Z(X)) \subset Z\left(X^{* *}\right)$ which is valid for arbitrary Banach spaces $X\left(T \in Z(X) \Rightarrow T^{*} \in C\left(X^{*}\right) \Rightarrow T^{* *} \in C_{\infty}\left(X^{* *}\right)=Z\left(X^{* *}\right)\right.$ by Theorems 3.13 and 5.7 of [2]), we arrive at the desired result.

4.4. TheOREM. Let $X$ be an $M$-ideal in $X^{* *}$ without proper $M$-summands. Then every nontrivial $M$-ideal of $X^{* *}$ contains $X$.

Proof. Let $J$ be an $M$-ideal in $X^{* *}$. Then $J \cap X$ is an $M$-ideal in $X$, hence by Theorem 4.1 an $M$-summand. The assumption then gives that $J \cap X$ equals $\{0\}$ or $X$. Assume the first case. By [2, 2.7 and 2.8(ii)] $J$ and $X$ are complementary $M$ summands in $J+X$. But this implies $J=\{0\}$, because there are no $M$-orthogonal directions for $X$ in $X^{* *}$. (Proof. Suppose that for $y \in X^{* *} \backslash X$ we have $\operatorname{span}(y, X)=\mathbf{K} y \oplus_{\infty} X$. Then $P_{X}(y)$, the set of best approximants of $y$ in $X$, is a closed ball in $X$ with positive radius $d(y, X)$. But as is easily seen, cf. [19]: For an arbitrary Banach space $X$ and $y \in X^{* *} \backslash X, P_{X}(y)$ has no interior points relative to $X$.)

Remarks. (1) The above result contains Theorem 3.4 of Smith and Ward [21] as a special case; cf. also [6]. (Note that by Proposition 5.7 below $K\left(l_{p}\right)$ does not contain proper $M$-summands.)

(2) In contrast to the situation $X=K\left(l_{p}\right), 1<p<\infty$, where Smith and Ward have shown in [22] that $X$ is the only nontrivial $M$-ideal in $X^{* *}$, in general there might exist $M$-ideals strictly between $X$ and $X^{* *}$.

EXAMPLE. For arbitrary Hilbert spaces $H, K(H)$ is an $M$-ideal in its bidual $L(H)$. The centralizer of a $W^{*}$-algebra is the centre of this algebra, so that by Theorem 4.3 $K(H)$ has a trivial centralizer. Denote by $\delta(X)$ the density character of the space $X$ (i.e. the smallest cardinal number, such that there exists a subset of this cardinality dense in $X$ ). It is not hard to show that for $\alpha \geqslant \boldsymbol{\kappa}_{0}$

$$
I_{\alpha}=\{T \in L(H) \mid \delta(\operatorname{im} T) \leqslant \alpha\}
$$

is a closed two-sided ideal in $L(H)$. By [20, Theorem 5.3] it is an $M$-ideal, so that for $H$ nonseparable we have e.g. $K(H) \varsubsetneqq I_{\aleph_{0}} \subsetneq L(H)$.

Note. This also provides easy examples of Banach spaces $X$ with trivial centralizer and arbitrary many $M$-ideals (take $X=L(H)$ with $H$ sufficiently large).

(3) By using the above results, it is possible to give a new (and shorter) proof of Theorem 3 of Lima [13]: If $X^{*} \cong L^{1}(\mu)$, then $X$ is an $M$-ideal in $X^{* *} \Leftrightarrow X \cong c_{0}(I)$, $I$ discrete.

This result shows that the converse of Corollary 3.2 is not valid in general. Take e.g. $X=c$. Then $X^{*} \cong l_{1}$ is an $L$-summand in $X^{* * *}$, but $X$, since not isometrically isomorphic to $c_{0}$, is not an $M$-ideal in $X^{* *}$.

(4) As a nice consequence we obtain the following "renorming" result: $d\left(X, c_{0}\right)=1 \Rightarrow X \cong c_{0}$. (Proof. The assumption obviously implies $d\left(X^{* *}, l_{\infty}\right)=1$, and this is known to imply $X^{* *} \cong l_{\infty}$. By the uniqueness of the predual of $l_{\infty}$, we get $X^{*} \cong l_{1}$, i.e. $X$ is an $L^{1}$-predual space. From Remark (3) following Theorem 3.4 we know that $X$ is an $M$-ideal in $X^{* *}$. Hence we get $X \cong c_{0}$.) 
5. Spaces of compact operators. A very difficult problem is to characterize those spaces $X$ such that $K(X)$ is an $M$-ideal in $L(X)$. This has been considered in many papers: e.g. Hennefeld [8, 9], Lima [13, 14] and Smith and Ward [20]. In [14] it is shown that if $K(X)$ is an $M$-ideal in $L(X)$, then $X$ is an $M$-ideal in $X^{* *}$. As there are reflexive spaces $X$ for which $K(X)$ is not an $M$-ideal in $L(X)$ (cf. [13]) the converse is not true. Here we show the following: If $X$ is reflexive and $K(X)$ is an $M$-ideal in $L(X)$, then $K(X)^{* *} \cong L(X)$, i.e. $K(X)$ is an $M$-ideal in its bidual. In order to obtain this result, we prove the existence of a bounded net of compact operators which converges in the strong operator topology to the identity. In the second half of this section we investigate the $M$-summands in $K(X, Y)$ : it is shown that only if $X^{*}$ or $Y$ have proper $M$-summands, $K(X, Y)$ can have nontrivial ones.

5.1. Lemma. Assume $K(X)$ is an $M$-ideal in $L(X)$. Then there exists a net $\left(T_{\alpha}\right)$ in $K(X)$ such that:

(i) $\left\|T_{\alpha}\right\| \leqslant 1$ for all $\alpha$.

(ii) $\left\|T_{\alpha} x-x\right\| \rightarrow 0$ for all $x \in X$.

(iii) $\left\|T_{\alpha}^{*} f-f\right\| \rightarrow 0$ for all $f \in X^{*}$.

(iv) $\left\|I-T_{\alpha}\right\| \rightarrow 1$.

Proof. As proved in [14], $X^{*}$ has the RNP. Let st.exp. $B_{X^{*}}$ denote the set of elements in the unit ball of $X^{*}$ which are strongly exposed by elements of $X^{* *}$. By [4], we have

$$
B_{X^{*}}=\overline{\operatorname{conv}}\left(\text { st.exp. } B_{X^{*}}\right) \text { (norm-closure). }
$$

Let $f_{1}, \ldots, f_{n} \in$ st.exp. $B_{X^{*}}$ and let $y_{1}, \ldots, y_{n} \in B_{X^{* *}}$ such that $1=\left\|y_{i}\right\|=y_{i}\left(f_{i}\right)$. Let $\partial>0$ and choose $\varepsilon>0$ such that if $g \in B_{X^{*}}$ with $y_{i}(g) \geqslant 1-\varepsilon$ for some $i$, then $\left\|g-f_{i}\right\|<\partial$. Choose $x_{i} \in X$ such that $\left\|x_{i}\right\|=1$ and $f_{i}\left(x_{i}\right)>1-\varepsilon / 2$. Define $S_{i} \in$ $K(X)$ by $S_{i}(x)=f_{i}(x) x_{i}$. Since $K(X)$ is an $M$-ideal in $L(X)$, there exists $T \in K(X)$ [11] such that

$$
T \in B(0,1) \cap B(I, 1+\partial) \cap \bigcap_{ \pm} \bigcap_{i=1}^{n} B\left(I \pm S_{i}, 1+\varepsilon / 2\right) .
$$

Thus $\|T\| \leqslant 1$ and $\|I-T\| \leqslant 1+\partial$. Since $S_{i}^{*}\left(f_{i}\right)=f_{i}\left(x_{i}\right) \cdot f_{i}$, we get

$$
\max _{ \pm}\left\|f_{i}\left(x_{i}\right) \cdot f_{i} \pm\left(f_{i}-T^{*} f_{i}\right)\right\| \leqslant 1+\varepsilon / 2
$$

such that

$$
\max _{ \pm}\left\|f_{i} \pm\left(f_{i}-T^{*} f_{i}\right)\right\| \leqslant 1+\varepsilon
$$

Hence

$$
\left|1 \pm\left(1-y_{i}\left(T^{*} f_{i}\right)\right)\right| \leqslant 1+\varepsilon
$$

such that $y_{i}\left(T^{*} f_{i}\right) \geqslant 1-\varepsilon$. But then $\left\|f_{i}-T^{*} f_{i}\right\|<\partial$.

Let $\Omega=$ the family of finite subsets of st.exp. $B_{X^{*}}$. We order $\Omega \times \mathbf{N}$ by $\left(\omega_{2}, n\right) \geqslant\left(\omega_{1}, m\right)$ if $\omega_{2} \supset \omega_{1}$ and $n \geqslant m$. We have now shown that for each $\alpha=$ $(\omega, n) \in \Omega \times \mathbf{N}$, there exists $T_{\alpha} \in K(X)$ such that $\left\|T_{\alpha}\right\| \leqslant 1$ and $\left\|T_{\alpha}^{*} f-f\right\|<1 / n$ if $f \in \omega$. 
Let $g \in X^{*}$, and assume $\|g\| \leqslant 1$. Let $\eta>0$. We can find $f_{1}, \ldots, f_{m} \in$ st.exp. $B_{X^{*}}$ such that

$$
d\left(g, \operatorname{conv}\left(f_{1}, \ldots, f_{m}\right)\right)<\eta / 3 .
$$

Let $n \in \mathbf{N}$ be such that $1 / n<\eta / 3$ and let $\omega=\left\{f_{1}, \ldots, f_{m}\right\}$ and $\alpha=(\omega, n)$. If now $T_{\alpha}$ is as above, then we easily see that $\left\|T_{\alpha}^{*} g-g\right\|<\eta$. Thus it follows that the net $\left(T_{\alpha}\right)$ satisfies (i), (iii) and (iv).

Clearly, $\left(T_{\alpha}\right)$ satisfies $f\left(T_{\alpha} x\right) \rightarrow f(x)$ for all $f \in X^{*}$ and all $x \in X$. Thus $T_{\alpha} x \rightarrow x$ weakly for all $x \in X$. By a well-known procedure, it follows that we can find a net $\left(T_{\alpha}^{\prime}\right)$ consisting of convex sums of the elements $\left(T_{\alpha}\right)$ which also satisfies (ii). The proof is complete.

REMARK. A shorter proof of Lemma 5.1, which is due to the referee, goes as follows: Write $L(X)^{*}=K(X)^{0} \oplus_{1} Y$, and let $z$ be the Hermitian projection in $L(X)^{* *}$ associated with $K(X)$. By Theorem 2.5 of [22], there exists a net $\left\{T_{\alpha}\right\}$ from $K(X)$ such that:

(1) $\left\|T_{\alpha}\right\| \rightarrow 1$.

(2) $\left\|I-T_{\alpha}\right\| \rightarrow 1$.

(3) $T_{\alpha} \rightarrow z\left(w^{*}\right.$ in $\left.L(X)^{* *}\right)$.

If $x \in X, f \in X^{*}$ and $F \in X^{* *}$, then the linear functionals on $L(X)$ defined by $T \rightarrow f(T x)$ and $T \rightarrow F\left(T^{*} x\right)$ are in $Y$ (since they attain their norms on $\left.K(X)\right)$. Since $I$ and $z$ agree on $Y$, we get by (3) that $T_{\alpha} x \rightarrow x$ weakly and $T_{\alpha}^{*} f \rightarrow f$ weakly for all $x \in X$ and all $f \in X^{*}$. By replacing the $T_{\alpha}$ 's by appropriate convex combinations, the lemma follows.

In [5], it is shown that the map $V: X^{*} \hat{\otimes}_{\pi} X^{* *} \rightarrow K(X)^{*}$ defined by

$$
\langle T, V(u)\rangle=\operatorname{tr}\left(T^{* *} u\right)=\sum_{i=1}^{\infty}\left\langle x_{i}^{*}, T^{* *} x_{i}^{* *}\right\rangle
$$

where $u=\sum_{i=1}^{\infty} x_{i}^{*} \otimes x_{i}^{* *} \in X^{*} \hat{\otimes}_{\pi} X^{* *}$, is a quotient map if $X^{*}$ has the RNP. It follows that we have natural identifications

$$
K(X)^{* *} \cong(\operatorname{ker} V)^{0} \subset\left(X^{*} \hat{\otimes}_{\pi} X^{* *}\right) * \cong L\left(X^{* *}\right) .
$$

5.2. Lemma. Assume $K(X)$ is an $M$-ideal in $L(X)$. If $T \in L(X)$, then $T^{* *} \in$ $(\operatorname{ker} V)^{0}$. Thus we have $L(X) \subset K(X)^{* *} \subset L\left(X^{* *}\right)$.

Proof. Note that by $\left(X^{*} \hat{\otimes}_{\pi} X^{* *}\right)^{*} \cong L\left(X^{* *}\right)$ an $S \in L\left(X^{* *}\right)$ acts on $u \in$ $X^{*} \hat{\otimes}_{\pi} X^{* *}$ by $u \mapsto \operatorname{tr}(S u):=\operatorname{tr}\left[\left(\operatorname{id}_{X^{*}} \otimes S\right) u\right]$. Let $T \in L(X)$, let $\left(T_{\alpha}\right) \subset K(X)$ be as in the lemma above and let $u=\sum_{i=1}^{\infty} x_{i}^{*} \otimes x_{i}^{* *} \in \operatorname{ker} V$. Then $T_{\alpha} T \in K(X)$ such that

$$
\begin{aligned}
0 & =\left\langle T_{\alpha} T, V(u)\right\rangle=\sum_{i=1}^{\infty}\left\langle x_{i}^{*}, T_{\alpha}^{* *} T^{* *} x_{i}^{* *}\right\rangle \\
& =\sum_{i=1}^{\infty}\left\langle T_{\alpha}^{*} x_{i}^{*}, T^{* *} x_{i}^{* *}\right\rangle \rightarrow \sum_{i=1}^{\infty}\left\langle x_{i}^{*}, T^{* *} x_{i}^{* *}\right\rangle=\operatorname{tr}\left(T^{* *} u\right) .
\end{aligned}
$$

Thus $T^{* *} \in(\operatorname{ker} V)^{0}$. 
5.3. TheOREM. If $X$ is reflexive and $K(X)$ is an $M$-ideal in $L(X)$, then $L(X)$ is isometrically isomorphic to $K(X)^{* *}$.

Proof. This is an easy consequence of Lemma 5.2.

A simple application of Corollary 3.7 to the embedding $X^{*} \rightarrow K(X)$ gives a partial converse of this theorem.

5.4. Proposition. If $K(X)$ is an $M$-ideal in $K(X)^{* *}$, then $X$ is reflexive.

A simple consequence of Lemma 5.1 and the fact that there exists a subspace of $l_{p}$ without the compact approximation property [15, Theorem II.1.g.4] is the following result.

5.5. Corollary. Let $1 \leqslant p \leqslant \infty, p \neq 2$. There exists a subspace $X$ of $l_{p}$ such that $K(X)$ is not an M-ideal in $L(X)$.

We now turn to the $M$-summands in $K(X, Y)$. Note that if there are proper $M$-summands in $X^{*}$ or $Y, K(X, Y)$ also has nontrivial ones (cf. [12, Propositions 6.1 and 6.3]). The next theorem gives the converse.

5.6. Theorem. Let $X$ and $Y$ be real Banach spaces. If $K(X, Y)$ contains a proper $M$-summand, then $Y$ contains a proper $M$-summand or $X$ contains a proper L-summand.

Proof. Several times we will use the following result of Ruess and Stegall [17]:

$$
S:=\operatorname{ext} B_{K(X, Y)^{*}}=\operatorname{ext} B_{X^{* *}} \otimes \operatorname{ext} B_{Y^{*}}
$$

where for $x^{* *} \in \operatorname{ext} B_{X^{* *}}, y^{*} \in \operatorname{ext} B_{Y^{*}}$ and $T \in K(X, Y)$

$$
\left(x^{* *} \otimes y^{*}\right)(T)=y^{*}\left(T^{* *} x^{* *}\right) .
$$

Now for a nontrivial $M$-projection $P$ in $K(X, Y), P^{*}$ is an $L$-projection in $K(X, Y)^{*}$ and $P \neq 0, I$. With Theorem 4.6 of [12] and the above result there exist $x_{0}^{* *}$, $x_{1}^{* *} \in \operatorname{ext} B_{X^{* *}}$ and $y_{0}^{*}, y_{1}^{*} \in \operatorname{ext} B_{Y^{*}}$ with $P^{*}\left(x_{0}^{* *} \otimes y_{0}^{*}\right)=0$ and $P^{*}\left(x_{1}^{* *} \otimes y_{1}^{*}\right)=$ $x_{1}^{* *} \otimes y_{1}^{*} \cdot x_{0}^{* *} \otimes y_{1}^{*}$ then belongs to $S$ and we have (i) $P^{*}\left(x_{0}^{* *} \otimes y_{1}^{*}\right)=x_{0}^{* *} \otimes y_{1}^{*}$ or (ii) $P^{*}\left(x_{0}^{* *} \otimes y_{1}^{*}\right)=0$.

ad (i). We claim that in this case $Y$ contains a proper $M$-summand.

Proof. Choose $f \in X^{*}$ with $x_{0}^{* *}(f)=1$. For each $y \in Y$, define $T_{y} \in K(X, Y)$ by $T_{1} z=f(z) y, z \in X$. Then $T_{y}^{* *} x_{0}^{* *}=y$. Now define $R: Y^{*} \rightarrow Y^{*}$ by

$$
\left(R y^{*}\right)(y)=\left[\left(P T_{y}\right) * * x_{0}^{* *}\right]\left(y^{*}\right)=\left(x_{0}^{* *} \otimes y^{*}\right)\left(P T_{y}\right)=\left[P^{*}\left(x_{0}^{* *} \otimes y^{*}\right)\right]\left(T_{y}\right) .
$$

As is easily seen, $R$ is a well-defined linear operator. Because $P T_{y}$ is compact, we know $\left(P T_{y}\right)^{* *} x_{0}^{* *} \in Y$; for a $w^{*}$-convergent net $\left(y_{\alpha}^{*}\right)$ in $Y^{*}$ with limit $y^{*}$ we thus have

$$
\left(R y_{\alpha}^{*}\right)(y)=y_{\alpha}^{*}\left[\left(P T_{y}\right)^{* *} x_{0}^{* *}\right] \rightarrow y^{*}\left[\left(P T_{y}\right)^{* *} x_{0}^{* *}\right]=\left(R y^{*}\right)(y)
$$

which shows that $R$ is $w^{*}-w^{*}$-continuous, hence of the form $R=Q^{*}$ for some $Q \in L(Y)$. We claim that $Q$ is a nontrivial $M$-projection.

Note first that we have

$$
R y^{*}=0 \text { or } y^{*} \text { for } y^{*} \in \operatorname{ext} B_{Y^{*}}
$$


(Proof.

$$
\begin{array}{rlrl}
\left(R y^{*}\right)(y) & =\left[P^{*}\left(x_{0}^{* *} \otimes y^{*}\right)\right]\left(T_{y}\right) & \\
& =0\left(T_{y}\right) \quad \text { or } \quad\left(x_{0}^{* *} \otimes y^{*}\right)\left(T_{y}\right) & & \text { because } x_{0}^{* *} \otimes y^{*} \in S \\
& \left.=0 \quad \text { or } y^{*}\left(T_{y}^{* *} x_{0}^{* *}\right)=y^{*}(y) .\right) & & \text { and } P^{*} \text { is an } L \text {-projection }
\end{array}
$$

But (*) gives $R=R^{2}$ on ext $B_{Y^{*}}$, hence on span ext $B_{Y^{*}}$. The $w^{*}$-density of this space and the $w^{*}$-continuity of $R$ show that $R$ is a projection, hence also $Q$ is a projection. By Theorem 4.6 of [12] and (*) $Q$ is already an $M$-projection. $P^{*}\left(x_{0}^{* *} \otimes y_{0}^{*}\right)=0$ and $P^{*}\left(x_{0}^{* *} \otimes y_{1}^{*}\right)=x_{0}^{* *} \otimes y_{1}^{*}$ imply (see the proof of $(*)$ ) $R y_{0}^{*}=0$ and $R y_{1}^{*}=y_{1}^{*}$, i.e. $Q$ is nontrivial and (i) is settled.

ad (ii). We claim that in this case $X^{*}$ contains a proper $M$-summand. (Note that $X$ then contains a proper $L$-summand by [2, Theorem 5.7].)

As the proof of this claim is similar, we omit the details. Choose $y \in Y$ with $y_{1}^{*}(y)=1$. For each $x^{*} \in X^{*}$ define $T_{x^{*}} \in K(X, Y)$ by $T_{x^{*}} z=x^{*}(z) y, z \in X$. Then $T_{x^{*}}^{*} y_{1}^{*}=x^{*}$. Now define $R: X^{* *} \rightarrow X^{* *}$ by

$$
\left(R x^{* *}\right)\left(x^{*}\right)=\left[\left(P T_{x^{*}}\right)^{* *} x^{* *}\right]\left(y_{1}^{*}\right)=\left(x^{* *} \otimes y_{1}^{*}\right)\left(P T_{x^{*}}\right)=\left[P^{*}\left(x^{* *} \otimes y_{1}^{*}\right)\right]\left(T_{x^{*}}\right) \text {. }
$$

$R$ is a nontrivial $w^{*}-w^{*}$-continuous $L$-projection.

As a corollary of the proof we obtain the following

5.7. Proposition. Let $X$ be a reflexive real Banach space. If $K(X)$ contains $a$ proper $M$-ideal, then $K(X)$ and $\left(X\right.$ or $\left.X^{*}\right)$ contain proper $M$-summands.

Proof. Let $M$ be a nontrivial $M$-ideal in $K(X)$ and $P$ the corresponding $L$-projection in $K(X)^{*}$ with range $M^{0}$. Because there are extreme points in the unit balls of $M^{0}$ and $M^{0 \perp} \cong M^{*}$, we can derive from $K(X)^{*}=M^{0} \oplus_{1} M^{0 \perp}$ the existence of $x_{0}^{* *}, x_{1}^{* *} \in \operatorname{ext} B_{X^{* *}}$ and $y_{0}^{*}, y_{1}^{*} \in \operatorname{ext} B_{Y^{*}}$ with the same properties as in the proof of the theorem, where $P^{*}$ is now replaced by $P$.

Assume we are in case (i). Let $T_{y}$ be as above and define $R: X^{*} \rightarrow X^{*}$ by

$$
\left(R y^{*}\right)(y)=\left[P\left(x_{0}^{* *} \otimes y^{*}\right)\right]\left(T_{y}\right) .
$$

$R$ is a well-defined linear operator and, since $X$ is reflexive, a dual operator. Hence, as before, $R$ is a nontrivial $L$-projection. Thus $X$ contains a proper $M$-summand. (ii) is similar and both cases clearly imply that $K(X)$ contains a proper $M$-summand, cf. [12, Propositions 6.1 and 6.3].

REMARK. Together with Theorem 4.4, the above results contain Theorem 3.2 of [21] as a special case.

\section{REFERENCES}

1. T. Ando, On the predual of $H^{\infty}$, Special issue dedicated to L. Orlicz, Comment. Math. Special Issue 1 (1978), 33-40.

2. E. Behrends, M-structure and the Banach-Stone-Theorem, Lecture Notes in Math., vol. 736, Springer-Verlag, Berlin, 1979.

3. E. Behrends et al., $L^{P}$-structure in real Banach spaces, Lecture Notes in Math., vol. 613, Springer-Verlag, Berlin, 1977. 
4. J. Diestel and J. J. Uhl, Vector measures, Math. Surveys, no. 15, Amer. Math. Soc., Providence. R. I., 1977.

5. M. Feder and P. Saphar, Spaces of compact operators and their dual spaces, Israel J. Math. 21 (1975). $38-49$.

6. P. Flinn, $A$ characterization of $M$-ideals in $B\left(l^{P}\right)$ for $1<p<\infty$, Pacific J. Math. 98 (1982), 73-80.

7. G. Godefroy, Espace de Banach: Existence et unicite de certains preduaux, Ann. Inst. Fourier (Grenoble) 28 (1978), 87-105.

8. J. Hennefeld, $A$ decomposition of $B(X)^{*}$ and unique Hahn-Banach extensions. Pacific J. Math. 46 (1973), 197-199.

9. M-ideals, HB-subspaces, and compact operators, Indiana Univ. Math. J. 28 (1979). 927-934.

10. R. B. Holmes, Geometric functional analysis and its applications, Springer-Verlag. Berlin, 1975.

11. A. Lima, Intersection properties of balls and subspaces in Banach spaces, Trans. Amer. Math. Soc. 227 (1977), 1-62.

12. Intersection properties of balls in spaces of compact operators, Ann. Inst. Fourier (Grenoble) 28 (1978), 35-65.

13. __ M-ideals of compact operators in classical Banach spaces, Math. Scand. 44 (1979), 207-217.

14. __ On M-ideals and best approximation, Indiana Univ. Math. J. 31 (1982), 27-36.

15. J. Lindenstrauss and L. Tzafriri, Classical Banach spaces. I, II, Springer-Verlag, Berlin, 1977, 1979.

16. H. P. Rosenthal, On relative disjoint families of measures, Studia Math. 37 (1970), 13-36, 311-313.

17. W. Ruess and C. Stegall, Extreme points in duals of operator spaces. Math. Ann. 261 (1982), 535-546.

18. K. Saatkamp, M-ideals of compact operators, Math. Z. 158 (1978), 253-263.

19. Schnitteigenschaften und Beste Approximation, Dissertation, Bonn, 1979.

20. R. R. Smith and J. D. Ward, M-ideal structure in Banach algebras. J. Funct. Anal. 27 (1978), $337-349$.

21. _ M-ideals in $B\left(I^{P}\right)$, Pacific J. Math. 81 (1979), 227-237.

22. Applications of convexity and M-ideal theory to quotient Banach algebras, Quart. J. Math. Oxford Ser. (2) 30 (1979), 365-384.

23. M. Takesaki, On the conjugate space of operator algebras. Tôhoku Math. J. 10 (1958). 194-203.

Institut für Mathematik I, Freie Universität Berlin, Arnimallee 3, D-1000 Berlin 33. GerMany

Department of Mathematics and Statistics, Agricultural University of Norway, 1432 AAS - NLH, NORWAY 